\title{
Modeling and Robust Discrete LQ Repetitive Control of Electrically Driven Robots
}

\author{
Mohammad Mehdi Fateh Maryam Baluchzadeh \\ Department of Electrical and Robotic Engineering, Shahrood University of Technology, Shahrood 3619995161, Iran
}

\begin{abstract}
Discrete linear quadratic control has been efficiently applied to linear systems as an optimal control. However, a robotic system is highly nonlinear, heavily coupled and uncertain. To overcome the problem, the robotic system can be modeled as a linear discrete-time time-varying system in performing repetitive tasks. This modeling motivates us to develop an optimal repetitive control. The contribution of this paper is twofold. For the first time, it presents discrete linear quadratic repetitive control for electrically driven robots using the mentioned model. The proposed control approach is based on the voltage control strategy. Second, uncertainty is effectively compensated by employing a robust time-delay controller. The uncertainty can include parametric uncertainty, unmodeled dynamics and external disturbances. To highlight its ability in overcoming the uncertainty, the dynamic equation of an articulated robot is introduced and used for the simulation, modeling and control purposes. Stability analysis verifies the proposed control approach and simulation results show its effectiveness.
\end{abstract}

Keywords: Discrete linear quadratic control, repetitive control, electrically driven robot, robust time-delay controller, uncertainty.

\section{Introduction}

Industrial robots perform repetitive tasks in many manufacturing applications. Repetitive control is a promising control approach to achieve tracking of the periodic trajectories. This kind of control has gained a great deal of research interest in various forms, such as passivitybased repetitive control ${ }^{[1]}$, nonlinear repetitive control ${ }^{[2]}$, time-delay repetitive control ${ }^{[3]}$, minimum-norm and timeoptimal repetitive control ${ }^{[4]}$, optimal repetitive control ${ }^{[5]}$ and adaptive repetitive control ${ }^{[6]}$.

Industrial robots meet key structural features to perform repetitive trajectories in many manufacturing applications. Therefore, model-based control can be used for tracking repetitive trajectories if a precise model is available. Some discrete models, such as the one presented in [7], are too complex, computationally extensive and impractical in real time control. On the other hand, simplified discrete models employed in the digital control ${ }^{[8]}$ may produce errors. The discrete linear control methods are degraded due to using imprecise models. Among the linear discrete repetitive model-based control algorithms for robot manipulators, i.e., Q-filter, convolution, learning and basis function, the Q-filter algorithm shows the fastest execution speed, the lowest computational complexity, and ease of design and implementation ${ }^{[9]}$. However, its tracking performance is not satisfactory.

It has been found that a robotic system can be modeled as a linear discrete-time time-varying system ${ }^{[4]}$. This modeling motivates us to efficiently apply the optimal control to robot manipulators if the uncertainty can be well compensated. The contribution of this paper is twofold. First, a discrete linear quadratic repetitive control for electrically driven robots is developed using a linear discrete time-varying model. The novel control approach is based on the voltage control strategy by considering the whole robotic system including the robot manipulator and its joint motors. Second, the uncertainty is effectively compensated by employing a robust time-delay controller.

The majority of the developed control approaches for the robot manipulators are based on the torque control strategy so far. The torque control approaches may have some shortcomings. This type of control is nonlinear, coupled and computationally extensive due to the characteristics of the robot dynamics. In addition, the dynamics of the robot's motors are excluded from the control problem. To remove these shortcomings, the voltage control strategy has been developed $^{[10]}$. So far, robust voltage control for electrically driven robots has been presented in various approaches, such as fuzzy control ${ }^{[11]}$, tasks-pace control ${ }^{[12]}$, time-delay control $^{[13]}$, adaptive control ${ }^{[14]}$, repetitive control ${ }^{[4]}$ and robust control by adaptive fuzzy estimation of uncertainty ${ }^{[15]}$.

The rest of this paper is organized as follows. Section 2 introduces the linear discrete-time time-varying model of the robot manipulator. Section 3 develops the repetitive discrete linear quadratic control including the robust time-delay controller and the discrete linear quadratic controller. Section 4 presents the stability analysis. Section 5 illustrates the simulation results and presents comparisons. Finally, Section 6 concludes the paper.

\section{$2 \quad$ Modeling}

The discrete linear quadratic (DLQ) control of robot faces a difficulty that a model of robot is highly nonlinear, heavily coupled and uncertain. However, a robot can be represented in the form of linear discrete-time time-varying system $^{[4]}$ as follows. The dynamics of a robotic system driven by permanent magnet direct current (DC) motors in continuous time ${ }^{[16]}$ can be expressed as 


$$
\begin{aligned}
& R_{a} K_{m}^{-1}\left(J_{m} r^{-1}+r D(q)\right) \ddot{q}+ \\
& \quad\left(R_{a} K_{m}^{-1} B_{m} r^{-1}+R_{a} K_{m}^{-1} r C(q, \dot{q})+K_{b} r^{-1}\right) \dot{q}+ \\
& \quad R_{a} K_{m}^{-1} r g(q)+d+\xi=V
\end{aligned}
$$

where $q \in \mathbf{R}^{n}$ is the vector of generalized joint positions, $D(q)$ is the $n \times n$ inertia matrix, $C(q, \dot{q}) \dot{q} \in \mathbf{R}^{n}$ is the vector of generalized centripetal and Coriolis forces, and $g(q) \in \mathbf{R}^{n}$ is the vector of generalized gravitational forces. The $n \times n$ positive diagonal coefficient matrices $J_{m}, B_{m}, \quad r, K_{m}, K_{b}$ and $R_{a}$ are the inertia, damping, reduction gear ratio, torque constant, back EMF constant, and resistance of motors, respectively. $V \in \mathbf{R}^{n}$ represents a vector of motor voltages as the input of the robotic system. $d \in \mathbf{R}^{n}$ stands for the effect of motor inductances and $\xi \in \mathbf{R}^{n}$ presents the external disturbances. This model is highly nonlinear, heavily coupled, multivariable and uncertain.

Equality (1) can be rewritten as

$$
M(q) \ddot{q}+N(q, \dot{q}) \dot{q}+W(q)+\xi=V
$$

where

$$
\begin{aligned}
& M(q)=R_{a} K_{m}^{-1}\left(J_{m} r^{-1}+r D(q)\right) \\
& N(q, \dot{q})=\left(R_{a} K_{m}^{-1} B_{m}+R_{a} K_{m}^{-1} r^{2} C(q, \dot{q})+K_{b}\right) r^{-1} \\
& W(q)=R_{a} K_{m}^{-1} r g(q) .
\end{aligned}
$$

Then, it is easy to show that

$$
\begin{aligned}
\ddot{q}=- & M^{-1}(q) N(q, \dot{q}) \dot{q}-M^{-1}(q) W(q)- \\
& M^{-1}(q) \xi+M^{-1}(q) V(t) .
\end{aligned}
$$

Using nominal terms in (6), we can obtain that

$$
\begin{gathered}
\ddot{q}=-\hat{M}^{-1}(q) \hat{N}(q, \dot{q}) \dot{q}-\hat{M}^{-1}(q) \hat{W}(q)+ \\
\hat{M}^{-1}(q) V(t)+\phi
\end{gathered}
$$

where $\hat{M}(q), \hat{N}(q, \dot{q})$ and $\hat{W}(q)$ are the nominal terms for the real terms $M(q), N(q, \dot{q})$ and $W(q)$, and $\phi$ is the lumped uncertainty.

It is assumed that the nominal terms are given with the same dynamics as the real terms with parametric errors. One can notice this assumption and propose a nominal model used for designing the controller. Therefore, the following assumption is made.

Assumption 1. The nominal terms $\hat{M}(q), \hat{N}(q, \dot{q})$ and $\hat{W}(q)$ are given with the same dynamics as the real terms $M(q), N(q, \dot{q})$ and $W(q)$, respectively. In other words, they face only the parametric uncertainty.

The lumped uncertainty $\phi$ is expressed by substituting (6) into (7) as

$$
\begin{aligned}
\phi= & \left(\hat{M}^{-1}(q) \hat{N}(q, \dot{q})-M^{-1}(q) N(q, \dot{q})\right) \dot{q}+ \\
& \hat{M}^{-1}(q) W(q)-M^{-1}(q) W(q)-M^{-1}(q) \xi+ \\
& \left(M^{-1}(q)-M^{-1}(q)\right) V(t) .
\end{aligned}
$$

The lumped uncertainty $\phi$ includes the parametric uncertainty, unmodeled dynamics and external disturbances.
Assume that there exists a $V_{d}(t)$ which satisfies

$$
\begin{aligned}
\ddot{q}_{d}= & -\hat{M}^{-1}\left(q_{d}\right) N\left(q_{d}, \dot{q}_{d}\right) \dot{q}_{d}- \\
& \hat{M}^{-1}\left(q_{d}\right) W\left(q_{d}\right)+M^{-1}\left(q_{d}\right) V_{d}(t)
\end{aligned}
$$

where $q_{d}$ is the desired trajectory. Thus, $V_{d}(t)$ can be calculated from (9) by using the desired trajectory as

$$
V_{d}(t)=\hat{M}\left(q_{d}\right) \ddot{q}_{d}+N\left(q_{d}, \dot{q}_{d}\right) \dot{q}_{d}+W\left(q_{d}\right) .
$$

Subtracting (7) from (9) yields

$$
\begin{aligned}
\ddot{q}_{d}-\ddot{q}= & \hat{M}^{-1}\left(q_{d}\right) V_{d}(t)-M^{-1}(q) V(t)+ \\
& \hat{M}^{-1}(q) N(q, \dot{q}) \dot{q}-\hat{M}^{-1}\left(q_{d}\right) N\left(q_{d}, \dot{q}_{d}\right) \dot{q}_{d}+ \\
& \hat{M}^{-1}(q) W(q)-\hat{M}^{-1}\left(q_{d}\right) W\left(q_{d}\right)-\phi
\end{aligned}
$$

or

$$
\begin{aligned}
\ddot{q}_{d}-\ddot{q}= & -\hat{M}^{-1}\left(q_{d}\right) N\left(q_{d}, \dot{q}_{d}\right)\left(\dot{q}_{d}-\dot{q}\right)+ \\
& M^{-1}\left(q_{d}\right)\left(V_{d}(t)-V(t)\right)+M^{-1}\left(q_{d}\right) \rho
\end{aligned}
$$

where uncertainty $\rho$ is expressed as

$$
\begin{aligned}
\rho= & \left(\hat{M}\left(q_{d}\right) M^{-1}(q) \hat{N}(q, \dot{q})-N\left(q_{d}, \dot{q}_{d}\right)\right) \dot{q}- \\
& \left(\hat{M}\left(q_{d}\right) M^{-1}(q)-I\right) V(t)-M\left(q_{d}\right) \phi+ \\
& \hat{M}\left(q_{d}\right) M^{-1}(q) \hat{W}(q)-W\left(q_{d}\right)
\end{aligned}
$$

and $I$ is the identify matrix.

Using (12), the state space equation is given as

$$
\dot{X}=A\left(q_{d}, \dot{q}_{d}\right) X+B\left(q_{d}\right) u+B\left(q_{d}\right) \rho
$$

where $X$ is the state vector, $u$ the input vector, $A\left(q_{d}, \dot{q}_{d}\right)$ is the state matrix, and $B\left(q_{d}\right)$ is a gain matrix. The details are

$$
\begin{aligned}
& A\left(q_{d}, \dot{q}_{d}\right)=\left[\begin{array}{ll}
0 & I \\
0 & -\hat{M}^{-1}\left(q_{d}\right) N\left(q_{d}, \dot{q}_{d}\right)
\end{array}\right] \\
& X=\left[\begin{array}{c}
q_{d}-q \\
\dot{q}_{d}-\dot{q}
\end{array}\right] \\
& B\left(q_{d}\right)=\left[\begin{array}{l}
0 \\
\hat{M}^{-1}\left(q_{d}\right)
\end{array}\right] \\
& u=V_{d}-V .
\end{aligned}
$$

The proposed model in (14) has an advantage that $A\left(q_{d}, \dot{q}_{d}\right)$ and $B\left(q_{d}\right)$ are known in advance. However, this model includes uncertainty $\rho$. The proposed model is an uncertain linear time-variant system with periodical matrices denoted by $A\left(q_{d}, \dot{q}_{d}\right)$ and $B\left(q_{d}\right)$. In fact, $A\left(q_{d}, \dot{q}_{d}\right)$ and $B\left(q_{d}\right)$ are period functions since $q_{d}$ and $\dot{q}_{d}$ are periodic functions of time due to performing repetitive task.

From (14), one can obtain a linear discrete system using a sampling period $\sigma$, which is a small positive constant. Substituting $k \sigma$ into $t$ for $k=1,2, \cdots$, and then approximating $\dot{X}$ as $\dot{X}=\frac{X(t+\sigma)-X(t)}{\sigma}$ provides a discrete model of the form

$$
x_{k+1}=A_{k} x_{k}+B_{k} u_{k}+B_{k} \rho_{k}
$$

where $x_{k}=X(k \sigma), A_{k}=I+\sigma A(\sigma k), B_{k}=\sigma B(\sigma k)$, $u_{k}=u(\sigma k)$ and $\rho_{k}$ denotes the uncertainty. Since $A_{k}$ and $B_{k}$ are available, they can be computed in advance. 


\section{Repetitive DLQ control}

The DLQ control has been efficiently applied to certain linear systems as an optimal control. In order to employ the DLQ control for the robotic system (15), the nonlinearities and uncertainties must be compensated.

Some assumptions and conditions are given to design the robust controller. To make the dynamics of tracking error well-defined such that the robot can track the desired trajectory, we make the following assumptions.

Assumption 2. The desired trajectory $q_{d}$ must be smooth in the sense that $q_{d}$ and its derivatives up to a necessary order are available and all uniformly bounded.

Smoothness of the desired trajectory can be guaranteed by proper trajectory planning.

As a necessary condition to design a robust controller, the matching condition must be satisfied: The uncertainty must enter the system through the same channel as the control input. Then, the uncertainty is said to satisfy the matching condition ${ }^{[17]}$ or is equivalently said to be matched. Fortunately, the matching condition is satisfied by the robot manipulator ${ }^{[18]}$. In system (14), the lumped uncertainty $\rho$ and the control input $u$ enter the system through the same channel.

As a necessary condition to design a robust control, the external disturbance $\xi$ in (1) must be bounded.

Assumption 3. The external disturbance $\xi$ is bounded as

$$
\|\xi\| \leqslant \xi_{\max }
$$

where $\xi_{\max }$ is a positive constant.

The voltage of every motor should be limited to protect the motor against over voltage. For this purpose, every motor is equipped with a voltage limiter. Therefore, we introduce Assumption 4.

Assumption 4. The motor voltages are bounded as

$$
\|V\| \leqslant V_{\max }
$$

where $V_{\max }$ is a positive constant.

A two-term control law is proposed to track the repetitive trajectory. The first term is the DLQ controller and the second term is a robust controller.

The system is then presented as

$$
x_{k+1}=A_{k} x_{k}+B_{k} u_{1, k}+B_{k} u_{2, k}+B_{k} \rho_{k}
$$

where $u_{1, k}$ and $u_{2, k}$ are the first and second terms of the control input. Performance of the repetitive control is improved if the lumped uncertainty $\rho_{k}$ is compensated.

\subsection{Robust time-delay controller}

In order to estimate and compensate the uncertainty, a robust time-delay controller is used to compensate the uncertainty. This type of uncertainty estimation was successfully used to estimate the uncertainty in the robust impedance control of a hydraulic suspension system ${ }^{[19]}$, the robust control of flexible-joint robots ${ }^{[13]}$, and minimumnorm and time-optimal repetitive control ${ }^{[4]}$.
Considering (19), the uncertainty $\rho_{k}$ can be perfectly compensated if

$$
B_{k} u_{2, k}=-B_{k} \rho_{k} .
$$

Since $\rho_{k}$ is not known, control law (19) cannot be defined. Thus, uncertainty $\rho_{k}$ should be estimated. To estimate uncertainty $\rho_{k}$, one can obtain from (19) that

$$
B_{k} \rho_{k}=x_{k+1}-A_{k} x_{k}-B_{k} u_{1, k}-B_{k} u_{2, k} .
$$

Since $x_{k+1}$ is not available in the $k$-th step, $B_{k} \rho_{k}$ cannot be calculated from (21). Using (21) in the previous step, one can write

$$
B_{k-1} \rho_{k-1}=x_{k}-A_{k-1} x_{k-1}-B_{k-1} u_{1, k-1}-B_{k-1} u_{2, k-1} .
$$

The term $B_{k-1} \rho_{k-1}$ can be calculated since all terms in the right hand side of (22) are known. The estimation of the uncertainty $B_{k} \rho_{k}$ as $B_{k-1} \rho_{k-1}$ is the basis of the robust time-delay control law:

$$
B_{k} u_{2, k}=-B_{k-1} \rho_{k-1} .
$$

The time-delay controller can be calculated by substituting (22) into (23) as

$$
B_{k} u_{2, k}=-x_{k}+A_{k-1} x_{k-1}+B_{k-1} u_{1, k-1}+B_{k-1} u_{2, k-1} .
$$

Thus,

$$
\begin{aligned}
u_{2, k}= & \left(B_{k}^{\mathrm{T}} B_{k}\right)^{-1} B_{k}^{\mathrm{T}} \times \\
& \left(-x_{k}+A_{k-1} x_{k-1}+B_{k-1} u_{1, k-1}+B_{k-1} u_{2, k-1}\right) .
\end{aligned}
$$

In order to evaluate the estimation of $B_{k} \rho_{k}$ as $B_{k-1} \rho_{k-1}$, the following explanation is given. $B$ is a continuous function as defined in (15). Therefore, $B_{k}$ is roughly the same as $B_{k-1}$. If the uncertainty is smooth, $\rho_{k}$ can be well approximated as $\rho_{k-1}$. Otherwise, there is a difference between $\rho_{k}$ and $\rho_{k-1}$. From (21) and (22), it can be written that

$$
\begin{aligned}
& B_{k} \rho_{k}-B_{k-1} \rho_{k-1}= \\
& \quad x_{k+1}-x_{k}+A_{k-1} x_{k-1}-A_{k} x_{k}+ \\
& \quad B_{k-1} u_{1, k-1}-B_{k} u_{1, k}+B_{k-1} u_{2, k-1}-B_{k} u_{2, k} .
\end{aligned}
$$

Since the state vector $x(t)$ and state matrix $A(t)$ are continuous,

$$
x_{k+1}-x_{k}+A_{k-1} x_{k-1}-A_{k} x_{k} \approx 0
$$

Thus,

$$
\begin{aligned}
& B_{k} \rho_{k}-B_{k-1} \rho_{k-1} \approx \\
& B_{k-1} u_{1, k-1}+B_{k-1} u_{2, k-1}-B_{k} u_{1, k}-B_{k} u_{2, k} .
\end{aligned}
$$
by

Using $u=u_{1}+u_{2}$, the estimation error can be calculated

$$
B_{k} \rho_{k}-B_{k-1} \rho_{k-1} \approx B_{k-1} u_{k-1}-B_{k} u_{k} .
$$

The estimation error is dependent on the control input. Unless the control input jumps, the value of $B_{k-1} u_{k-1}-$ $B_{k} u_{k}$ is ignorable. 
The performance of the time-delay controller can be evaluated by substituting (20) into system (19).

As a result, the uncertainty is compensated as

$$
x_{k+1}=A_{k} x_{k}+B_{k} u_{1, k}+B_{k} \rho_{k}-B_{k-1} \rho_{k-1} .
$$

According to (29), $B_{k} \rho_{k}-B_{k-1} \rho_{k-1}$ is ignorable unless the control input jumps. Thus, system (30) can be well approximated to

$$
x_{k+1}=A_{k} x_{k}+B_{k} u_{1, k} .
$$

\subsection{DLQ controller}

The DLQ controller is given by

$$
u_{1, k}=-K_{k} x_{k}
$$

The gain matrix $K_{k}$ is calculated by minimizing a given cost function ${ }^{[20]}$ written in the set of real numbers as

$$
\begin{aligned}
L= & 0.5 x_{N}^{\mathrm{T}} S x_{N}+0.5 \sum_{k=0}^{N-1}\left(x_{k}^{\mathrm{T}} Q x_{k}+u_{1, k}^{\mathrm{T}} R u_{1, k}\right)+ \\
& \lambda_{k+1}^{\mathrm{T}}\left(A_{k} x_{k}+B_{k} u_{1, k}-x_{k+1}\right)
\end{aligned}
$$

with respect to $x_{k}, u_{1, k}$ and $\lambda_{k}$, where $\lambda_{k}$ is the Lagrange multiplier, $Q, S$ and $R$ are symmetric positive definite matrices. As a result, we have

$$
K_{k}=\left[R+B_{k}^{*} p_{k} B_{k}\right]^{-1} B_{k}^{*} p_{k} A_{k}
$$

where $p_{k}$ is calculated as

$$
\begin{aligned}
p_{k}= & Q+A_{k}^{*} p_{k-1} A_{k}- \\
& A_{k}^{*} p_{k-1} B_{k}\left[R+B_{k}^{*} p_{k-1} B_{k}\right]^{-1} B_{k}^{*} p_{k-1} A_{k} .
\end{aligned}
$$

The final control law is formed by substituting (24) and (32) into $u_{k}=u_{1, k}+u_{2, k}$ as

$$
\begin{aligned}
u_{k}= & \left(B_{k}^{\mathrm{T}} B_{k}\right)^{-1} B_{k}^{\mathrm{T}} \times \\
& \left(A_{k-1} x_{k-1}-\left(I+B_{k} K_{k}\right) x_{k}+B_{k-1} u_{1, k-1}\right) .
\end{aligned}
$$

The algorithm starts from $k=0$ in (35), where $p_{-1}=0$. Then, $K_{k}$ is calculated from (34). After that, $u_{0}$ is calculated from (36), in which $x_{0}$ is the initial value of state, $x_{-1}$ is given as $x_{-1}=x_{0}$ and $u_{-1}=0$.

\section{Stability analysis}

Applying the control laws (23) and (32) to system (16) results in the closed-loop system

$$
x_{k+1}=\left(A_{k}-B_{k} K_{k}\right) x_{k}+B_{k} \rho_{k}-B_{k-1} \rho_{k-1} .
$$

The boundedness of system states $q$ and $\dot{q}$ can verify the stability. This proof is presented as follows.

Proof. According to a proof given by [13], under Assumptions 3 and 4 , the joint velocities $\dot{q}$ and joint accelerations $\ddot{q}$ are bounded. It follows from Assumption 2 that the desired joint positions $q_{d}$, the desired joint velocities $\dot{q}_{d}$, and joint accelerations $\ddot{q}_{d}$ are bounded. According to the properties of robot manipulator ${ }^{[18]}$, the inertia matrix
$D(q)$ and the gravitational forces $g(q)$ are bounded. Because $\dot{q}$ is bounded, the Coriolis and centripetal term $C(q, \dot{q})$ is bounded ${ }^{[18]} . N(q, \dot{q})$ and $W(q)$ expressed in (3) - (5) are bounded since $J_{m}, B_{m}, r, K_{m}, K_{b}$ and $R_{a}$ are constant, and $D(q), C(q, \dot{q})$ and $g(q)$ are bounded. According to Assumption $1, \hat{M}(q), \hat{N}(q, \dot{q})$ and $\hat{W}(q)$ are bounded follows the boundedness of $D(q), C(q, \dot{q})$ and $g(q)$.

The boundedness of $\dot{q}, \ddot{q}, \dot{q}_{d}, \ddot{q}_{d}, M(q), N(q, \dot{q}), W(q)$, $\hat{M}(q), \hat{N}(q, \dot{q}), \hat{W}(q), \hat{M}\left(q_{d}\right), \hat{N}\left(q_{d}, \dot{q}_{d}\right)$ implies that $\phi$ stated in (8) and the lumped uncertainty $\rho$ expressed in (13) are bounded. Thus,

$$
\|\rho\| \leqslant \rho_{\max }
$$

where $\rho_{\max }$ is a positive scalar.

Since the DLQ controller provides $K_{k}$ such that $A_{k}-$ $B_{k} K_{k}$ is Hurwitz, system (37) is stable. In addition, the term $B_{k} \rho_{k}-B_{k-1} \rho_{k-1}$ as an input to system (37) is bounded. Therefore, the linear system (37) provides a bounded output $x_{k+1}$ under the bounded input $B_{k} \rho_{k}-$ $B_{k-1} \rho_{k-1}$. The boundedness of $x$ means the boundedness of $q_{d}-q$ and $\dot{q}_{d}-\dot{q}$. The boundedness of $q_{d}$ in Assumption 3 and the boundedness $q_{d}-q$ yields the boundedness of $q$.

As a result, the stability is proven due to the boundedness of system states denoted by $q$ and $\dot{q}$.

The control performance is evaluated as follows. According to the reasoning given above, the tracking error $q_{d}-q$ and its derivative $\dot{q}_{d}-\dot{q}$ are bounded. The robust timedelay controller (24) has a main role in compensating the uncertainty, thereby improving the control performance. If the uncertainties and nonlinearities cannot be well compensated, the closed-loop system (37) is subject to a large uncertainty. The residual uncertainty in the closed-loop system (37) has been reduced from a large value of $B_{k} \rho_{k}$ to a small value of $B_{k} \rho_{k}-B_{k-1} \rho_{k-1}$ due to using the robust time-delay control law (24). As a result, the control performance is well improved by reducing the residual uncertainty. The residual uncertainty $B_{k} \rho_{k}-B_{k-1} \rho_{k-1}$ will be very small when the uncertainty is smooth and the sampling time is very short.

\section{Simulation results}

A symbolic scheme of an articulated robot based on the Denavit-Hartenberg (DH) representation is shown in Fig. 1. The Denavit-Hartenberg parameters of the articulated robot are given in Table 1 , where parameters $\theta_{i}$, $d_{i}, a_{i}$ and $\alpha_{i}$, for $i=1, \cdots, n$, are called the joint angle, link offset, link length and link twist, respectively. Using the modeling approach presented in [21], we obtain the dynamical model of the articulated robot expressed in (1) as follows.

Matrices $J_{m}, B_{m}, r, K_{m}$ and $R_{a}$ are $3 \times 3$ positive diagonal coefficient matrices of the inertia, damping, reduction gear ratio, torque constant, and resistance of motors, respectively. In the diagonal matrices, the $i$-th component of the main diagonal shows the $i$-th motor coefficient. The details of matrices $D(q), C(q, \dot{q})$ and $G$ are given in the 
appendix, where

$$
\begin{aligned}
& D(q)=\left[\begin{array}{ccc}
D 11 & D 12 & D 13 \\
D 21 & D 22 & D 23 \\
D 31 & D 32 & D 33
\end{array}\right] \\
& C(q, \dot{q})=\left[\begin{array}{lll}
C 11 & C 12 & C 13 \\
C 21 & C 22 & C 23 \\
C 31 & C 32 & C 33
\end{array}\right] \\
& G=\left[\begin{array}{c}
G 1 \\
G 2 \\
G 3
\end{array}\right] .
\end{aligned}
$$

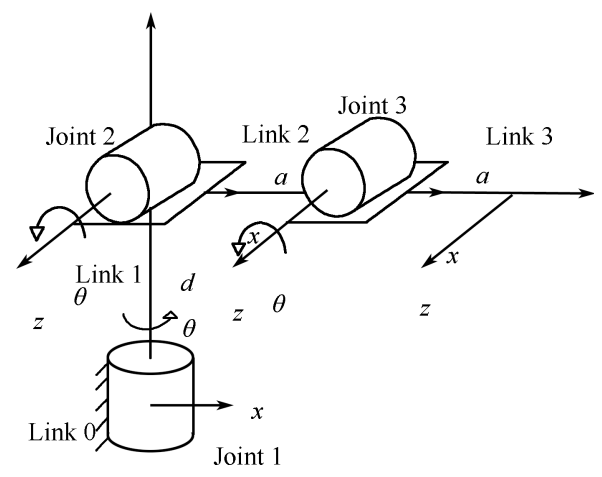

Fig. 1 The symbolic scheme of the articulated robot

Table 1 The Denavit-Hartenberg parameters

\begin{tabular}{ccccc}
\hline Link & $\theta$ & $d$ & $a$ & $\alpha$ \\
\hline 1 & $\theta_{1}$ & $d_{1}=0.28$ & $\frac{\pi}{2}$ & 0 \\
2 & $\theta_{2}$ & 0 & $a_{2}=0.76$ & 0 \\
3 & $\theta_{3}$ & 0 & $a_{3}=0.93$ & 0 \\
\hline
\end{tabular}

The dynamical parameters of manipulator are given in Table 2, in which $g=9.8 \mathrm{~m} / \mathrm{s}^{2}, q_{i}, i=1,2,3$ denote the joint angles, $m_{i}$ is the $i$-th link mass, $r_{i}=\left[\begin{array}{ccc}x_{i} & y_{i} & z_{i}\end{array}\right]^{\mathrm{T}}$ is the center of mass in the $i$-th frame, and the inertia tensor of the $i$-th link in the center of mass frame is given by

$$
I_{i}=\left[\begin{array}{ccc}
I x x_{i} & I y y_{i} & I x z_{i} \\
I x y_{i} & I y y_{i} & I y z_{i} \\
I x z_{i} & I y z_{i} & I z z_{i}
\end{array}\right]
$$

The motor parameters are given in Table 3 while the three motors are the same.

The discrete model of the robotic system is presented by (19). The DLQ controller is given by (32), (34) and (35). The robust time-delay controller is expressed by (25). The proposed control law including the DLQ controller and time delay controller is given by (36).

The inertia tensor in the centre of mass frame is expressed as $I x y_{i}=0, I x z_{i}=0, I y z_{i}=0, z_{i}=0$.
Table 2 Dynamical parameters of the articulated robot

\begin{tabular}{ccccccc}
\hline$i$ & $x_{i}$ & $y_{i}$ & $m_{i}$ & Ixx & Iyy & Izzi \\
\hline 1 & 0 & -0.22 & 19 & 0.34 & 0.36 & 0.31 \\
2 & -0.51 & 0 & 18.18 & 0.18 & 1.32 & 1.31 \\
3 & -0.67 & 0 & 10.99 & 0.07 & 0.92 & 0.93 \\
\hline
\end{tabular}

Table 3 Parameters of DC servomotors

\begin{tabular}{ccccccc}
\hline Motor & $K_{b}$ & $J_{m}$ & $B_{m}$ & $r$ & $R_{a}$ & $L_{a}$ \\
\hline $1,2,3$ & 0.26 & 0.0002 & 0.001 & 0.01 & 1.26 & 0.001 \\
\hline
\end{tabular}

The desired repetitive trajectory is given by

$$
q_{d}=\left[\begin{array}{lll}
\cos (0.1 \pi t) & \cos (0.1 \pi t) & \cos (0.1 \pi t)
\end{array}\right]^{\mathrm{T}}
$$

where $q_{d}$ is a vector of desired joint angles with a period of $20 \mathrm{~s}$

Simulations illustrate the performance of the proposed control law (36). The desired trajectory given by (41) is sufficiently smooth and the motors are sufficiently strong such that the robot can track the desired trajectory. We run the simulations for two periods to illustrate the repetitive motion.

The uncertainty may include the external disturbances, unmodeled dynamics, and parametric uncertainty. To consider the parametric uncertainty, all the parameters of the nominal model used in the control law are given as $95 \%$ of the real ones in Simulations 1-3. The effect of large parametric uncertainty is considered in Simulation 4, in which the nominal parameters are given as $70 \%$ of the real ones. The external disturbance is given to the input of each motor as a random signal with the maximum value $+4 \mathrm{~V}$ and the minimum value $-4 \mathrm{~V}$ with a period of $2 \mathrm{~s}$ as shown in Fig. 2 . The uncertainty is unknown. However, we have to use an example of a bounded uncertainty to check the performance of the control system.

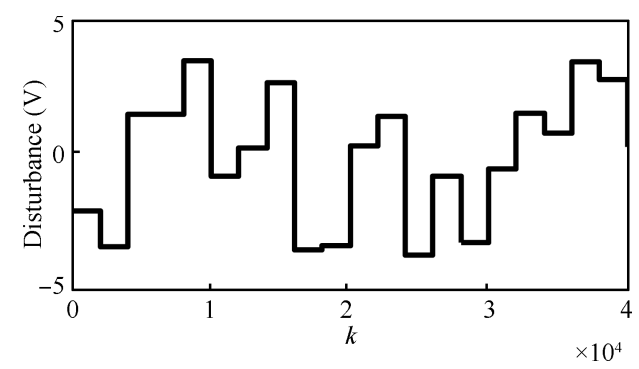

Fig. 2 Random disturbance

The matrices $Q$ and $R$ in (34) and (35) are given by trail and error method to have a good performance using $Q=10^{8} \times I_{6 \times 6}$ and $R=10 \times I_{3 \times 3}$, where $I_{n \times n}$ is the $n \times n$ identity matrix.

Simulation 1. The set point control is simulated. The initial positions of the joint angles are set to $q(0)=\left[\begin{array}{lll}0 \mathrm{rad} & 0.5 \mathrm{rad} 2 \mathrm{rad}\end{array}\right]$ while the initial position of the desired trajectory is given by $q_{d}(0)=$ 
[ $1 \mathrm{rad} 1 \mathrm{rad} 1 \mathrm{rad}]$. The initial error is calculated as $q_{d}(0)-q(0)=\left[\begin{array}{cc}1 \mathrm{rad} & 0.5 \mathrm{rad}-1 \mathrm{rad}\end{array}\right]$. As a practical regard, the motor voltages are limited to the maximum value of $40 \mathrm{~V}$ to protect the motors from over voltages. The task is repeated twice in $40 \mathrm{~s}$. The norm of tracking errors is vanished well after $5 \mathrm{~s}$ and comes under the $5 \times 10^{-6}$ rod at the end in Fig. 3. The motor voltages are under the permitted value of $40 \mathrm{~V}$ and behave well without any problems, as shown in Fig. 4. The jumps on the control efforts confirm that the control effort can promptly reply to the external disturbances. As a result, the uncertainties are compensated well.

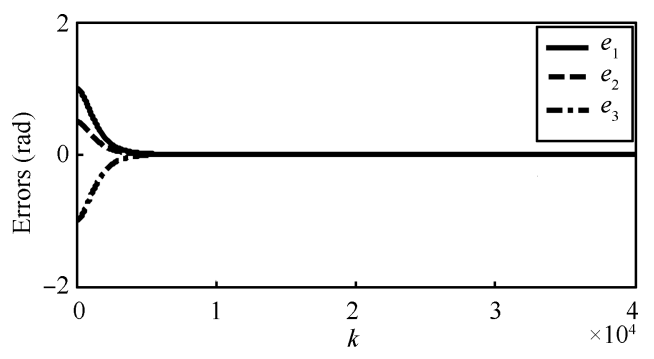

Fig. 3 Set point performance

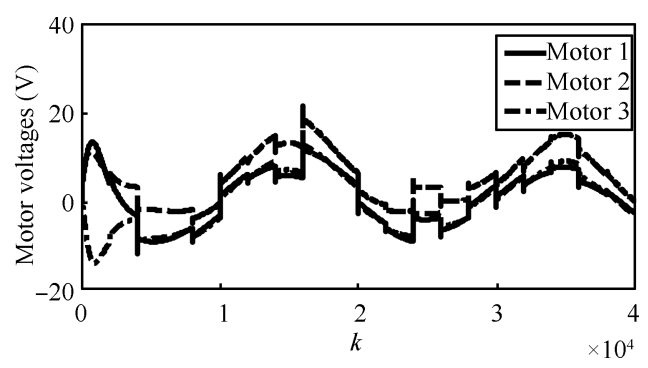

Fig. 4 Control efforts of the proposed control

Simulation 2. The tracking control is simulated. The initial error is given zero. The tracking error is ignorable with the maximum value of about $e_{1}=2.7 \times 10^{-5} \mathrm{rad}$, $e_{2}=0.5 \times 10^{-5} \mathrm{rad}$ and $e_{3}=5 \times 10^{-5} \mathrm{rad}$, as shown in Fig. 5. And the control efforts behave well under the permitted value of $40 \mathrm{~V}$ and promptly reply to the external disturbances as shown in Fig. 6.

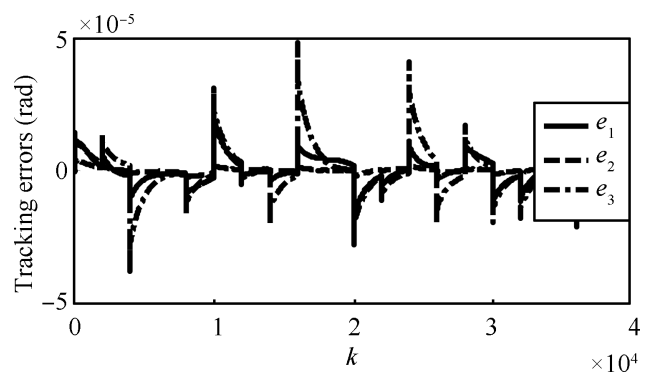

Fig. 5 Control performance without initial errors

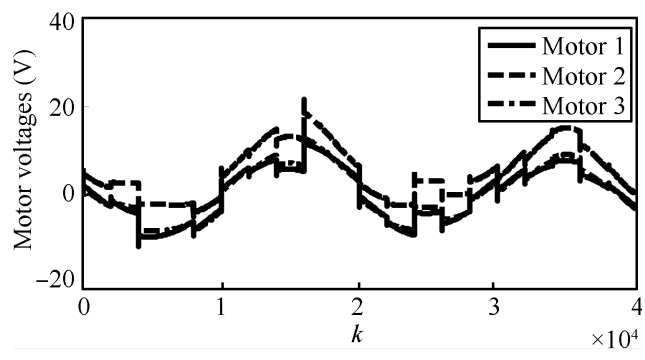

Fig. 6 Control efforts under zero initial errors

Simulation 3. The effect of the robust time-delay controller in compensating the uncertainty is evaluated in this simulation. For this purpose, the time-delay controller is removed. The initial error is given zero to highlight the accuracy of the tracking response. The tracking errors are shown in Fig. 7.

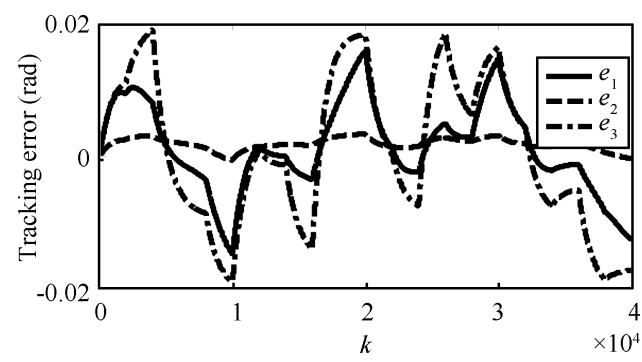

Fig. 7 Performance of the DLQ control

The maximum values of tracking errors are about $e_{1}=$ $0.01 \mathrm{rad}, e_{2}=0.03 \mathrm{rad}$ and $e_{3}=0.02 \mathrm{rad}$.

Compared to Simulation 2, the tracking errors are highly increased as the error for joint 2 is 600 times larger than the one in Simulation 2. The control efforts behave well under the permitted value of $40 \mathrm{~V}$ and promptly reply to the external disturbances as shown in Fig. 8. Comparing Figs. 5 and 7 confirms that the robust time-delay controller has significantly improved the tracking performance.

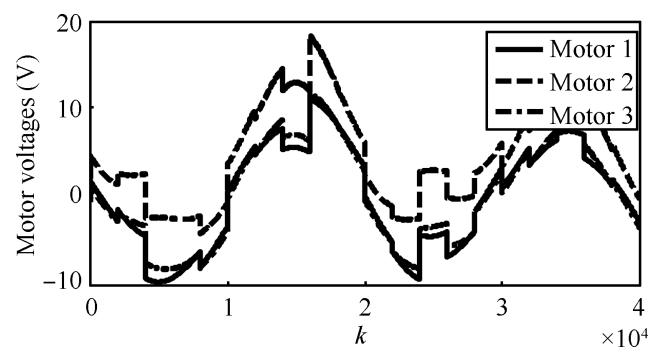

Fig. 8 Control efforts of DLQ under zero

Simulation 4. The effect of large parametric uncertainty is considered in this simulation. The nominal parameters are given as $70 \%$ of the real ones.

The set point performance is shown in Fig. 9. The control performance is similar to Simulation 1. The norm of error is vanished well after $5 \mathrm{~s}$ and comes under the $1 \times 10^{-5} \mathrm{rad}$ at the end, which is ignorable. 


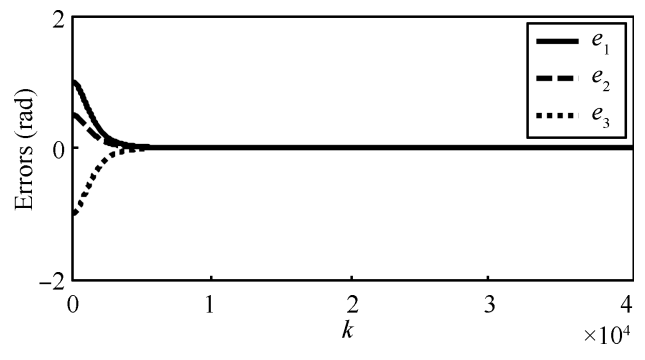

Fig. 9 Set point performance in large uncertainty

The tracking performance is shown in Fig. 10. The control performance is similar to Simulation 2. The tracking errors are ignorable with the maximum values of about $e_{1}=$ $4 \times 10^{-5} \mathrm{rad}$ and $e_{2}=2.75 \times 10^{-5} \mathrm{rad}$ and $e_{3}=6 \times 10^{-5} \mathrm{rad}$.

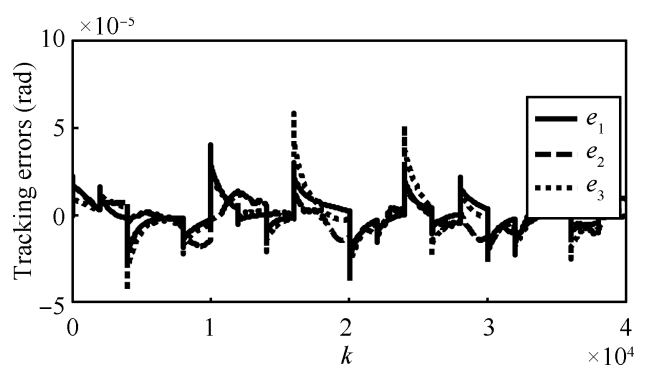

Fig. 10 Tracking performance in large uncertainty

The control performance is considered by removing the time-delay controller. The tracking errors are highly increased with the maximum values of about $e_{1}=0.023 \mathrm{rad}$, $e_{2}=0.025 \mathrm{rad}$ and $e_{3}=0.027 \mathrm{rad}$, as shown in Fig. 11 . However, the control performance is similar to Simulation 3.

It can be concluded that the control performance in large parametric errors is similar to the one in small parametric errors.

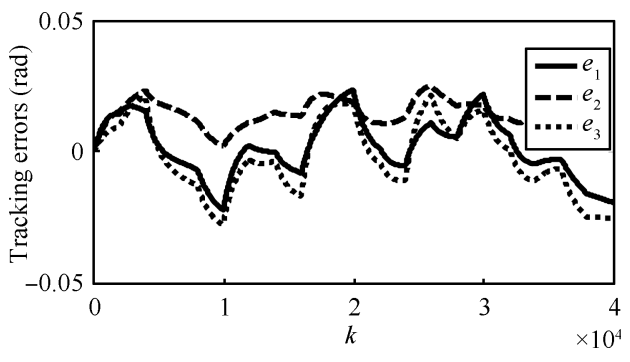

Fig. 11 Tracking performance without using the time-delay controller in large uncertainty

\section{Conclusions}

A novel robust optimal discrete repetitive control has been developed for electrically driven robot manipulators. A linear discrete-time and time-varying model has been used for the robotic system to apply the DLQ. As a result, an optimal control for a nonlinear system, such as a robot, is obtained. Then, the model uncertainty is efficiently compensated using a discrete robust time-delay controller. The robust controller estimates and compensates the uncertainty such that the use of nominal model becomes efficient. The robust controller has played an important role to improve the performance of the control system by reducing the residual uncertainty in the closed-loop system. Simulation results have shown a dominant role for the robust controller to provide a high-accuracy tracking performance. Despite many repetitive control approaches, the proposed control is able to compensate the non-repetitive errors and a wide range of uncertainties, including parametric errors, unmodeled dynamics and external disturbances. The stability of the control system is proven under the matching condition and Assumptions 1-3.

\section{Appendix}

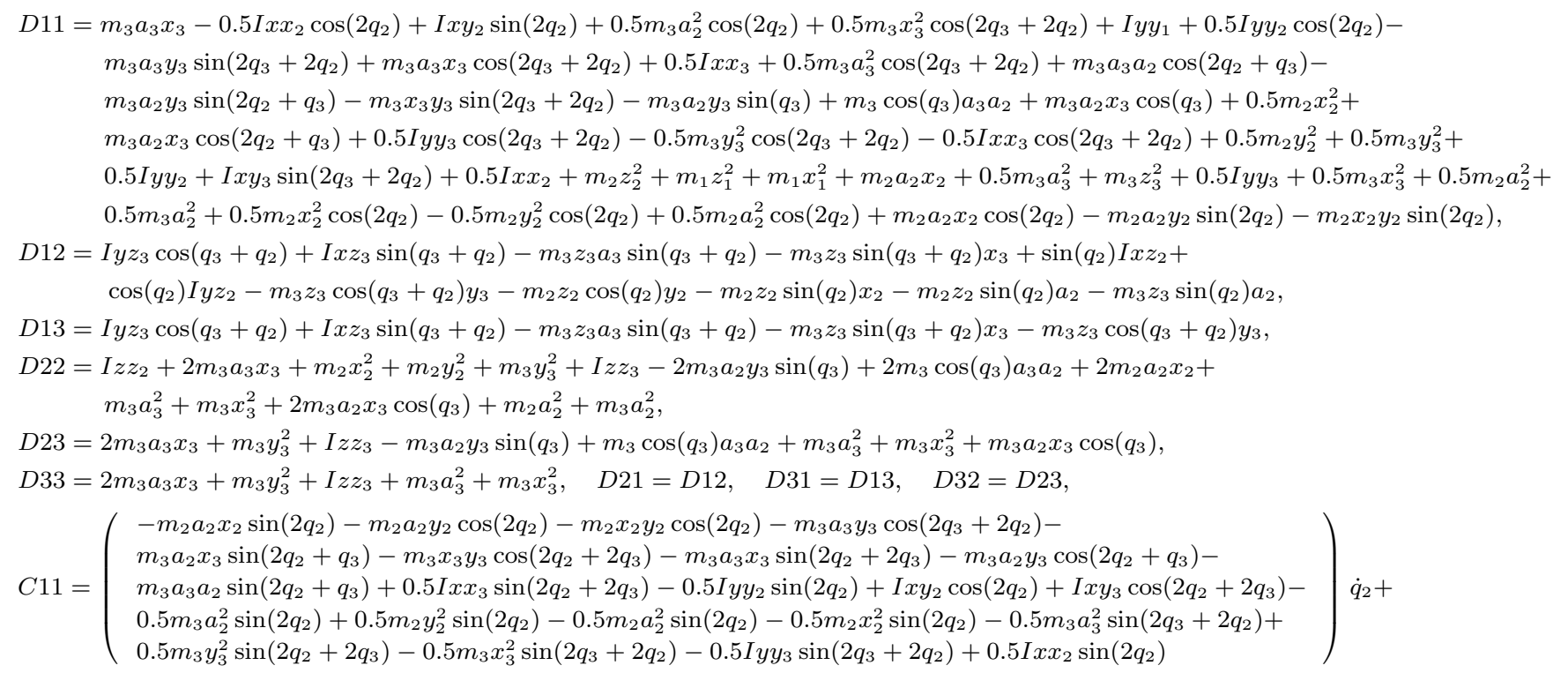




$$
\begin{aligned}
& \left(\begin{array}{l}
-0.5 m_{3} a_{2} y_{3} \cos \left(q_{3}\right)-0.5 m_{3} a_{2} x_{3} \sin \left(q_{3}\right)-m_{3} a_{3} y_{3} \cos \left(2 q_{2}+2 q_{3}\right)-0.5 m_{3} a_{2} x_{3} \sin \left(2 q_{2}+q_{3}\right)- \\
m_{3} x_{3} y_{3} \cos \left(2 q_{2}+2 q_{3}\right)-m_{3} a_{3} x_{3} \sin \left(2 q_{2}+2 q_{3}\right)-0.5 m_{3} a_{2} y_{3} \cos \left(2 q_{2}+q_{3}\right)-0.5 m_{3} a_{3} a_{2} \sin \left(2 q_{2}+q_{3}\right)- \\
0.5 m_{3} \sin \left(q_{3}\right) a_{3} a_{2}+0.5 I x x_{3} \sin \left(2 q_{2}+2 q_{3}\right)+I x y_{3} \cos \left(2 q_{2}+2 q_{3}\right)-0.5 m_{3} a_{3}^{2} \sin \left(2 q_{2}+2 q_{3}\right)+ \\
0.5 m_{3} y_{3}^{2} \sin \left(2 q_{2}+2 q_{3}\right)-0.5 m_{3} x_{3}^{2} \sin \left(2 q_{2}+2 q_{3}\right)-0.5 I y y_{3} \sin \left(2 q_{2}+2 q_{3}\right)
\end{array}\right) \dot{q}_{3}, \\
& C 12=\left(\begin{array}{l}
-m_{2} a_{2} x_{2} \sin \left(2 q_{2}\right)-m_{2} a_{2} y_{2} \cos \left(2 q_{2}\right)-m_{2} x_{2} y_{2} \cos \left(2 q_{2}\right)-m_{3} a_{3} y_{3} \cos \left(2 q_{3}+2 q_{2}\right)- \\
m_{3} a_{2} x_{3} \sin \left(2 q_{2}+q_{3}\right)-m_{3} x_{3} y_{3} \cos \left(2 q_{2}+2 q_{3}\right)-m_{3} a_{3} x_{3} \sin \left(2 q_{2}+2 q_{3}\right)-m_{3} a_{2} y_{3} \cos \left(2 q_{2}+q_{3}\right)- \\
m_{3} a_{3} a_{2} \sin \left(2 q_{2}+q_{3}\right)+0.5 I x x_{3} \sin \left(2 q_{2}+2 q_{3}\right)-0.5 I y y_{2} \sin \left(2 q_{2}\right)+I x y_{2} \cos \left(2 q_{2}\right)+I x y_{3} \cos \left(2 q_{2}+2 q_{3}\right)- \\
0.5 m_{3} a_{2}^{2} \sin \left(2 q_{2}\right)+0.5 m_{2} y_{2}^{2} \sin \left(2 q_{2}\right)-0.5 m_{2} a_{2}^{2} \sin \left(2 q_{2}\right)-0.5 m_{2} x_{2}^{2} \sin \left(2 q_{2}\right)-0.5 m_{3} a_{3}^{2} \sin \left(2 q_{3}+2 q_{2}\right)+ \\
0.5 m_{3} y_{3}^{2} \sin \left(2 q_{2}+2 q_{3}\right)-0.5 m_{3} x_{3}^{2} \sin \left(2 q_{3}+2 q_{2}\right)-0.5 I y y_{3} \sin \left(2 q_{3}+2 q_{2}\right)+0.5 I x x_{2} \sin \left(2 q_{2}\right)
\end{array}\right) \dot{q}_{1}+ \\
& \left(\begin{array}{l}
-m_{2} z_{2} \cos \left(q_{2}\right) x_{2}-m_{3} z_{3} a_{3} \cos \left(q_{3}+q_{2}\right)-m_{3} z_{3} \cos \left(q_{3}+q_{2}\right) x_{3}+m_{3} z_{3} \sin \left(q_{3}+q_{2}\right) y_{3}-I y z_{3} \sin \left(q_{3}+q_{2}\right)+ \\
I x z_{3} \cos \left(q_{3}+q_{2}\right)+m_{2} z_{2} \sin \left(q_{2}\right) y_{2}-m_{3} z_{3} \cos \left(q_{2}\right) a_{2}+\cos \left(q_{2}\right) I x z_{2}-\sin \left(q_{2}\right) I y z_{2}-m_{2} z_{2} \cos \left(q_{2}\right) a_{2}
\end{array}\right) \dot{q}_{2}+ \\
& \left(-m_{3} z_{3} a_{3} \cos \left(q_{3}+q_{2}\right)-m_{3} z_{3} \cos \left(q_{3}+q_{2}\right) x_{3}+m_{3} z_{3} \sin \left(q_{3}+q_{2}\right) y_{3}-I y z_{3} \sin \left(q_{3}+q_{2}\right)+I x z_{3} \cos \left(q_{3}+q_{2}\right)\right) \dot{q}_{3}, \\
& C 13=\left(\begin{array}{l}
-0.5 m_{3} a_{2} y_{3} \cos \left(q_{3}\right)-0.5 m_{3} a_{2} x_{3} \sin \left(q_{3}\right)-m_{3} a_{3} y_{3} \cos \left(2 q_{3}+2 q_{2}\right)-0.5 m_{3} a_{2} x_{3} \sin \left(2 q_{2}+q_{3}\right)- \\
m_{3} x_{3} y_{3} \cos \left(2 q_{3}+2 q_{2}\right)-m_{3} a_{3} x_{3} \sin \left(2 q_{3}+2 q_{2}\right)-0.5 m_{3} a_{2} y_{3} \cos \left(2 q_{2}+q_{3}\right)-0.5 m_{3} a_{3} a_{2} \sin \left(2 q_{2}+q_{3}\right)- \\
0.5 m_{3} \sin \left(q_{3}\right) a_{3} a_{2}+0.5 I x x_{3} \sin \left(2 q_{3}+2 q_{2}\right)+I x y_{3} \cos \left(2 q_{3}+2 q_{2}\right)-0.5 m_{3} a_{3}^{2} \sin \left(2 q_{3}+2 q_{2}\right)+ \\
0.5 m_{3} y_{3}^{2} \sin \left(2 q_{3}+2 q_{2}\right)-0.5 m_{3} x_{3}^{2} \sin \left(2 q_{3}+2 q_{2}\right)-0.5 I y y_{3} \sin \left(2 q_{3}+2 q_{2}\right)
\end{array}\right) \dot{q}_{1}+ \\
& \left(-m_{3} z_{3} a_{3} \cos \left(q_{3}+q_{2}\right)-m_{3} z_{3} \cos \left(q_{3}+q_{2}\right) x_{3}+m_{3} z_{3} \sin \left(q_{3}+q_{2}\right) y_{3}-I y z_{3} \sin \left(q_{3}+q_{2}\right)+I x z_{3} \cos \left(q_{3}+q_{2}\right)\right) \dot{q}_{2}+ \\
& \left(-m_{3} z_{3} a_{3} \cos \left(q_{3}+q_{2}\right)-m_{3} z_{3} \cos \left(q_{3}+q_{2}\right) x_{3}+m_{3} z_{3} \sin \left(q_{3}+q_{2}\right) y_{3}-I y z_{3} \sin \left(q_{3}+q_{2}\right)+I x z_{3} \cos \left(q_{3}+q_{2}\right)\right) \dot{q}_{3}, \\
& C 21=\left(\begin{array}{l}
m_{2} a_{2} x_{2} \sin \left(2 q_{2}\right)+m_{2} a_{2} y_{2} \cos \left(2 q_{2}\right)+m_{2} x_{2} y_{2} \cos \left(2 q_{2}\right)+m_{3} a_{3} y_{3} \cos \left(2 q_{2}+2 q_{3}\right)+ \\
m_{3} a_{2} x_{3} \sin \left(2 q_{2}+q_{3}\right)+m_{3} x_{3} y_{3} \cos \left(2 q_{2}+2 q_{3}\right)+m_{3} a_{3} x_{3} \sin \left(2 q_{2}+2 q_{3}\right)+m_{3} a_{2} y_{3} \cos \left(2 q_{2}+q_{3}\right)+ \\
m_{3} a_{3} a_{2} \sin \left(2 q_{2}+q_{3}\right)-0.5 I x x_{3} \sin \left(2 q_{2}+2 q_{3}\right)+0.5 I y y_{2} \sin \left(2 q_{2}\right)-I x y_{2} \cos \left(2 q_{2}\right)-I x y_{3} \cos \left(2 q_{2}+2 q_{3}\right)+ \\
0.5 m_{3} a_{2}^{2} \sin \left(2 q_{2}\right)-0.5 m_{2} y_{2}^{2} \sin \left(2 q_{2}\right)+0.5 m_{2} a_{2}^{2} \sin \left(2 q_{2}\right)+0.5 m_{2} x_{2}^{2} \sin \left(2 q_{2}\right)+0.5 m_{3} a_{3}^{2} \sin \left(2 q_{3}+2 q_{2}\right)- \\
0.5 m_{3} y_{3}^{2} \sin \left(2 q_{3}+2 q_{2}\right)+0.5 m_{3} x_{3}^{2} \sin \left(2 q_{3}+2 q_{2}\right)+0.5 I y y_{3} \sin \left(2 q_{3}+2 q_{2}\right)-0.5 I x x_{2} \sin \left(2 q_{2}\right)
\end{array}\right) \dot{q}_{1}, \\
& C 22=\left(-m_{3} \sin \left(q_{3}\right) a_{3} a_{2}-m_{3} a_{2} y_{3} \cos \left(q_{3}\right)-m_{3} a_{2} x_{3} \sin \left(q_{3}\right)\right) \dot{q}_{3}, \\
& C 23=\left(-m_{3} \sin \left(q_{3}\right) a_{3} a_{2}-m_{3} a_{2} y_{3} \cos \left(q_{3}\right)-m_{3} a_{2} x_{3} \sin \left(q_{3}\right)\right) \dot{q}_{2}+\left(-m_{3} \sin \left(q_{3}\right) a_{3} a_{2}-m_{3} a_{2} y_{3} \cos \left(q_{3}\right)-m_{3} a_{2} x_{3} \sin \left(q_{3}\right)\right) \dot{q}_{3}, \\
& C 31=\left(\begin{array}{l}
0.5 m_{3} a_{2} y_{3} \cos \left(q_{3}\right)+0.5 m_{3} a_{2} x_{3} \sin \left(q_{3}\right)+m_{3} a_{3} y_{3} \cos \left(2 q_{3}+2 q_{2}\right)+0.5 m_{3} a_{2} x_{3} \sin \left(2 q_{2}+q_{3}\right)+ \\
m_{3} x_{3} y_{3} \cos \left(2 q_{3}+2 q_{2}\right)+m_{3} a_{3} x_{3} \sin \left(2 q_{3}+2 q_{2}\right)+0.5 m_{3} a_{2} y_{3} \cos \left(2 q_{2}+q_{3}\right)+0.5 m_{3} a_{3} a_{2} \sin \left(2 q_{2}+q_{3}\right)+ \\
0.5 m_{3} \sin \left(q_{3}\right) a_{3} a_{2}-0.5 I x x_{3} \sin \left(2 q_{3}+2 q_{2}\right)-I x y_{3} \cos \left(2 q_{3}+2 q_{2}\right)+0.5 m_{3} a_{3}^{2} \sin \left(2 q_{3}+2 q_{2}\right)- \\
0.5 m_{3} y_{3}^{2} \sin \left(2 q_{3}+2 q_{2}\right)+0.5 m_{3} x_{3}^{2} \sin \left(2 q_{3}+2 q_{2}\right)+0.5 I y y_{3} \sin \left(2 q_{3}+2 q_{2}\right)
\end{array}\right) \dot{q}_{1}, \\
& C 32=\left(m_{3} \sin \left(q_{3}\right) a_{3} a_{2}+m_{3} a_{2} y_{3} \cos \left(q_{3}\right)+m_{3} a_{2} x_{3} \sin \left(q_{3}\right)\right) \dot{q}_{2}, \\
& C 33=0 \text {, } \\
& G 1=0 \text {, } \\
& G 2=g\left(\begin{array}{l}
m_{2} \cos \left(q_{2}\right) a_{2}+m_{2} \cos \left(q_{2}\right) x_{2}-m_{2} \sin \left(q_{2}\right) y_{2}+m_{3} a_{3} \cos \left(q_{3}+q_{2}\right)+m_{3} \cos \left(q_{2}\right) a_{2}+ \\
m_{3} \cos \left(q_{3}+q_{2}\right) x_{3}-m_{3} \sin \left(q_{3}+q_{2}\right) y_{3}
\end{array}\right), \\
& G 3=g m_{3}\left(a_{3} \cos \left(q_{3}+q_{2}\right)+\cos \left(q_{3}+q_{2}\right) x_{3}-\sin \left(q_{3}+q_{2}\right) y_{3}\right) \text {. }
\end{aligned}
$$

\section{References}

[1] J. Kasac, B. Novakovic, D. Majetic, D. Brezak. Passive finite-dimensional repetitive control of robot manipulators. IEEE Transactions on Control Systems Technology, vol. 16, no. 3, pp. 570-576, 2008.

[2] X. Tang, K. M. Tsang, W. L. Chan. Active power filter using nonlinear repetitive controller. International Journal of Control, Automation and Systems, vol. 9, no. 1, pp. 132$138,2011$.

[3] J. Na, R. Griñó, R. Costa-Castelló, X. Ren, Q. Chen. Repetitive controller for time-delay systems based on disturbance observer. IET Control Theory ane Applications, vol. 4, no. 11, pp. 2391-2404, 2010.

[4] M. M. Fateh, H. A. Tehrani, S. M. Karbassi. Repetitive control of electrically driven robot manipulators. International Journal of Systems Science, vol. 44, no. 4, pp. 775-785, 2013.

[5] M. Wu, Y. H. Lan, J. H. She, Y. He, L. Xu. Optimal repetitive control based on two-dimensional model. International
Journal of Innovative Computing, Information and Control, vol. 8, no. 3, pp. 1897-1905, 2012.

[6] N. Sadegh, R. Horowitz, W. W. Kao, M. Tomizuka. A unified approach to the design of adaptive and repetitive controllers for robotic manipulators. ASME Journal of Dynamic Systems, Measurement, and Control, vol.112, no. 4, pp. 618-629, 1990.

[7] C. P. Neuman, V. D. Tourassis. Discrete dynamic robot models. IEEE Transactions on Systems, Man Cybernetics, vol. 15, no. 2, pp. 193-204, 1985.

[8] I. M. Y. Mareels, H. B. Penfold, R. J. Evans. Controlling nonlinear time-varying systems via Euler approximations. Automatica, vol. 28, no. 4, pp.681-696, 1992.

[9] S. Arimoto. Learning control theory for robotic motion. International Journal of Adaptive Control and Signal Processing, vol. 4, no. 6, pp. 543-564, 1990.

[10] M. M. Fateh. On the voltage-based control of robot manipulators. International Journal of Control, Automation, and Systems, vol. 6, no. 5, pp. 702-712, 2008. 
[11] M. M. Fateh. Robust fuzzy control of electrical manipulators. Journal of Intelligent and Robotic Systems, vol.60, no. 3-4, pp. 415-434, 2010.

[12] M. M. Fateh. Robust voltage control of electrical manipulators in task-space. International Journal of Innovative Computing, Information and Control, vol. 6, no. 6, pp. 26912700,2010

[13] M. M. Fateh. Robust control of flexible-joint robots using voltage control strategy. Nonlinear Dynamics, vol. 67, no. 2, pp. 1525-1537, 2012.

[14] M. M. Fateh. Nonlinear control of electrical flexible-joint robots. Nonlinear Dynamics, vol. 67, no. 4, pp. 2549-2559, 2012 .

[15] M. M. Fateh, S. Khorashadizadeh. Robust control of electrically driven robots by adaptive fuzzy estimation of uncertainty. Nonlinear Dynamics, vol. 69, no. 3, pp. 1465-1477, 2012.

[16] M. M. Fateh. Proper uncertainty bound parameter to robust control of electrical manipulators using nominal model. Nonlinear Dynamics, vol. 61, no. 4, pp. 655-666, 2010.

[17] M. J. Corless, G. Leitmann. Continuous state feedback guaranteeing uniform ultimate boundedness for uncertain dynamics systems. IEEE Transactions on Automatic Control, vol. 26, no. 5, pp. 1139-1144, 1981.

[18] Z. H. Qu, D. M. Dawson. Robust Tracking Control of Robot Manipulators, New York: IEEE Press, Inc., 1996.

[19] M. M. Fateh. Robust impedance control of a hydraulic suspension system. International Journal of Robust and Nonlinear Control, vol. 20, no. 8, pp. 858-872, 2010.
[20] K. Ogata. Discrete-time Control Systems, Upper Saddle River, NJ: Prentice-Hall, 1987.

[21] M. W. Spong, S. Hutchinson, M. Vidyasagar. Robot Modelling and Control, New York: John Wiley \& Sons, Inc. 2006

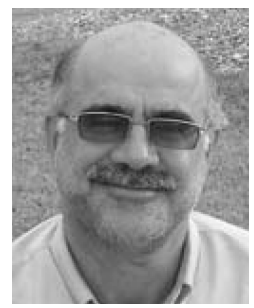

Mohammad Mehdi Fateh received his B. Sc. degree from Isfahan University of Technology in 1988, and his M. Sc. degree in electrical engineering from Tarbiat Modares University, Iran in 1991. He received his $\mathrm{Ph}$. $\mathrm{D}$. degree in robotic engineering from Southampton University, UK in 2001. He is a full professor with the Department of Electrical and Robotic Engineering, Shahrood University of Technology, Iran.

His research interests include nonlinear, robust and fuzzy control, robotics and intelligent systems, mechatronics and automation.

E-mail: mmfateh@shahroodut.ac.ir (Corresponding author)

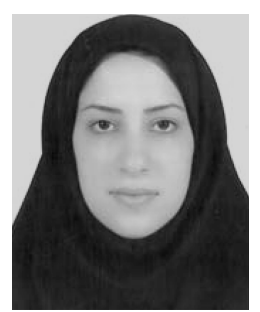

Maryam Baluchzadeh received her B. Sc. degree in control engineering from Ferdowsi University, Iran in 2010. She received her M. Sc. degree in control engineering at Shahrood University of Technology, Iran in 2012. Her thesis titled as Optimal Repetitive Control of Robot Manipulators Using Voltage Control Strategy was supervised by Professor Mohammad Mehdi Fateh.

Her research interests include robust control, discrete control and robotics.

E-mail: mbaluchzadeh85@yahoo.com 